\title{
Editorial
}

\section{The New System of Perinatal Care}

\section{Thomas Hulsey, MSPH, ScD}

Journal of Perinatology (2002) 22, 628-629 doi:10.1038/si.jp.7210855

In this issue of the Journal, Gould et al. describe the increase in the number of Level III hospitals, with the concomitant shift in patients, in California between 1990 and 1997. ${ }^{1}$ Most dramatically, community NICUs increased largely as a result of being redesignated, or formally acknowledged, as a higher-acuity hospital. In California, the number of VLBW in large regional centers declined 34\% (from 3044 to 2005) whereas the number of VLBW in community NICUs increased 182\% (from 1017 to 2866).

This paper serves as sobering evidence that traditional regionalized perinatal care, and much of the original guiding philosophy, have disappeared. The actions of the California hospitals and that of the California state agency that designates hospital level indicate that a new system of perinatal care has arrived. This new system has bluntly challenged apparently all the previously accepted performance standards.

Published reports that optimal VLBW outcomes are achieved in hospitals with large volume level III NICUs have been disputed. ${ }^{2}$ The old assumption that the health provider teams that cared for large numbers of high-risk patients were therefore more experienced and thus were able to obtain the optimal outcomes was also contested. In this study, there were substantial shifts in the location of delivery of VLBW newborns away from the large regional centers. This new system has also discounted the reasoning that the number of hospitals that are designated at each acuity level should in some way be reflective of the number of patients needing that particular level of care. In California, the number of VLBW births decreased by $9 \%$ whereas the number of NICUs increased by 19\%. There were 208 neonatologists in California in 1990 and by 1997, this had increased to 509 (nationally, this increase was from 1588 to 3688$)^{3}{ }^{3}$ This really should not come as much of a surprise since Pollack et al. found that nationally, the distribution of neonatologists had little to do with the distribution of very low birth weight births. ${ }^{4}$ This new system of perinatal care has additionally challenged the thought that an

Department of Pediatrics/Epidemiology, Medical University of South Carolina, Charleston, SC, USA.

Address correspondence and reprint requests to Thomas Hulsey, MSPH, ScD, Department of Pediatrics/Epidemiology, Medical University of South Carolina, PO Box 250566, Charleston, SC $29425-3313$, USA. efficient system of perinatal care is one where high-risk patients are consolidated into the minimum number of costly perinatal centers. In California, community NICUs increased from 17 to 52 to care for fewer VLBW births. These data provide dramatic evidence that the original tenets that guided the development of regionalized perinatal care are not contemporarily functional. Indeed, in California and elsewhere, the proliferation of neonatal intensive care programs has occurred in spite of published reports that such changes do not result in improved outcomes: either for the patient or the system of perinatal care. With supply (neonatologists) exceeding demand (high-risk newborns) and financial profitability an obvious certainty (otherwise why would so many hospitals be clamoring for level III status), more hospitals are entering the perinatal health care market place, regardless. If these new systems can redesign themselves regardless of what is published to the contrary and if these new systems have implications on perinatal health status or the cost of the system of care, then a new set of performance measures needs to be developed. What other measures should now be included to justify that these changes had a beneficial effect? A priori, what were the improvements that these changes were expected to achieve?

To begin with, how do these boutique community NICUs address the traditional regional center responsibilities of guaranteeing risk appropriate access to care regardless of ability to pay, outreach education activities, transport activities, assurance of access to high-risk follow-up programs for NICU graduates, tracking and surveillance activities of population health status, health care utilization, and outcomes? How do the populations served by the various centers differ with respect to payor classification, birth-weight-specific mortality and morbidity, and arrangements for access to primary care for mothers and babies after discharge?

Morbidity variation should include detailed information on markers such as: bronchopulmonary dysplasia, necrotising enterocolitis, intraventricular hemorrhage, periventricular leukomalasia, retinopathy of prematurity, nosocomial infection, seizures, pneumothorax, etc. Practice variation should include utilization parameters such as: length of time on ventilatory support, length of hospital stay, length of time to regain birth weight, and need for transport to another facility for specialized procedures (e.g., surgery).

Some measure of the overall systems performance of the new structure of perinatal health care should also be implemented. From a systems perspective, at the minimum there needs to be justification that the cost of the additional NICU beds and the cost of the additional neonatologists and other specialized health care personnel 
are reasonable, given some derived benefit gained from these added components. The cost benefit analysis needs clearer distinction. In California, more community hospitals now have neonatal intensive care units but the system has achieved the same outcomes, the demand for level III beds was basically unchanged, and the old resource structure could have accommodated the demand. The benefit to the newly designated intensive care programs is apparently revenue to the new NICUs - but at what cost to the overall system of perinatal health care? The additions of new designations of hospitals seem to fit well with a type of study design called the noninferiority trial.

This is where the new program does not claim to be better than the old program, just one that is not that much worse. In this situation, worse may be measured in increased costs. On the flip side, maybe the approach to modern newborn intensive care might be more appropriately viewed through the ecologic concept of "carrying capacity." For the perinatal health care system, this can be thought of as the maximum number of intensive care programs that can be sustained indefinitely in a given area. Or to put it another way, how many NICUs can be financially sustained given a rather stable number of high risk births each year? In California in 1990, the perinatal intensive care population was divided among 37 community plus regional NICUs. By 1997, the perinatal intensive care population of approximately the same size was divided among 82 community and regional NICUs.

How many NICUs can the California high-risk newborn population sustain? Apparently in California, the perinatal carrying capacity has not yet been reached. The perinatal health care system has now begun to look more like a traditional profit-oriented business. With little competition (as in regionalized perinatal care), a small group of companies (NICUs) might easily survive on the shared profits derived from the stable customer base (high-risk newborns).
With more companies entering the market, competition obviously becomes more aggressive because they are all seeking a share of a finite customer base. Economies of scale indicate that any company needs a certain level of customer base to remain profitable and in perinatal health care, since the carrying capacity of any region is fixed, there is clearly a limit to the number of companies that can be sustained. As the increased number of companies approaches a region's carrying capacity, in an attempt to maintain profitability, something must be compromised. Companies might cut prices (which never happens in health care), they may reduce costs by cutting certain services or quality, or they might rely on other product lines to cover the losses. Whatever the justifications for expansion, unbridled growth will eventually approach carrying capacity. Surveillance systems must be in place to assure that patient outcomes, quality of care, and the efficiency of the system are not those things that are compromised.

\section{To change and to change for the better are two different things — German Proverb.}

\section{References}

1. Gould JB, Marks AR, Chavez G. Expansion of community-based perinatal care in California. J Perinatol 2002;22(8):630-40.

2. Phibbs CS, Bronstein JM, Bixton E, Phibbs RH. The effects of patient volume and level of care at the hospital of birth on neonatal mortality. JAMA 1996;276(13):1054-9.

3. AAP. Department of Research, Division of Research on Health Policy. Physician Workforce Ratios for Child Health Care by County, HSA, State, U.S. Census Division (data from AMA: Physician Masterfile for 1990). Elk Grove Village, IL: AAP; 1993.

4. Pollack LD, Ratner IM, Lund GC. United States neonatology practice survey: personnel, practice, hospital, and neonatal intensive care unit characteristics. Pediatrics 1998;101(3):398-405. 